\section{Improving Iris Recognition Accuracy Via Cascaded Classifiers}

\author{
Zhenan Sun, Yunhong Wang, Tieniu Tan, and Jiali Cui
}

\begin{abstract}
As a reliable approach to human identification, iris recognition has received increasing attention in recent years. The most distinguishing feature of an iris image comes from the fine spatial changes of the image structure. So iris pattern representation must characterize the local intensity variations in iris signals. However, the measurements from minutiae are easily affected by noise, such as occlusions by eyelids and eyelashes, iris localization error, nonlinear iris deformations, etc. This greatly limits the accuracy of iris recognition systems. In this paper, an elastic iris blob matching algorithm is proposed to overcome the limitations of local feature based classifiers (LFC). In addition, in order to recognize various iris images efficiently a novel cascading scheme is proposed to combine the LFC and an iris blob matcher. When the LFC is uncertain of its decision, poor quality iris images are usually involved in intra-class comparison. Then the iris blob matcher is resorted to determine the input iris' identity because it is capable of recognizing noisy images. Extensive experimental results demonstrate that the cascaded classifiers significantly improve the system's accuracy with negligible extra computational cost.
\end{abstract}

Index Terms-Biometrics, blob matching, cascaded classifiers, iris recognition.

\section{INTRODUCTION}

With the increasing demand of enhanced security in our daily lives, reliable personal identification through biometrics is currently an active topic in the literature of pattern recognition. Biometric solutions, such as identification systems using fingerprint, iris, face, palmprint, etc., have many advantages over the traditional authentication techniques based on what you know or what you possess [1], [2]. Among them, iris recognition is tested as the most accurate manner of personal identification [3]. Therefore, nowadays many automatic security systems based on iris recognition have been deployed worldwide for border control, restricted access, and so on [4].

As seen in Fig. 1, the iris of the human eye is the annular part between the black pupil and the white sclera. It is clear that there are lots of irregular small blobs, such as freckles, coronas, stripes, furrows, crypts, etc., overlaying the iris region. Furthermore, the spatial distribution of these blocks in the iris is also random. Such randomly distributed and irregular blocks constitute the most distinguishing characteristics of the iris [5]. So how to analyze iris images or represent iris signals with symbols is a challenging problem.

For the last decade, a number of researchers have worked on iris recognition with the ambition to improve the application's performance specifications, such as accuracy, processing speed, storage cost, and robustness [6]-[24]. According to the various iris features utilized, these algorithms can be grouped into four main categories.

1) Phase-based method [6]-[10]: The phase is chosen as an iris feature because in 1981 Oppenheim and Lim [25] had demonstrated

Manuscript received March 15, 2004; revised May 5, 2004. This work is funded by research grants from the National Basic Research Program (Grant No. 2004CB318110), Natural Science Foundation of China (Grant No. 60335010, $60121302,60275003,60332010,69825105)$ and the Chinese Academy of Sciences. This paper was recommended by Guest Editor D. Zhang.

The authors are with the Center for Biometrics and Security Research, National Laboratory of Pattern Recognition, Institute of Automation, Chinese Academy of Sciences, Beijing, P.R. China 100080 (e-mail: znsun@nlpr.ia.ac.cn; wangyh@nlpr.ia.ac.cn; tnt@nlpr.ia.ac.cn; jlcui@nlpr.ia.ac.cn).

Y. Wang is also with Beihang University, Beijing 100083, China.

Digital Object Identifier 10.1109/TSMCC.2005.848169
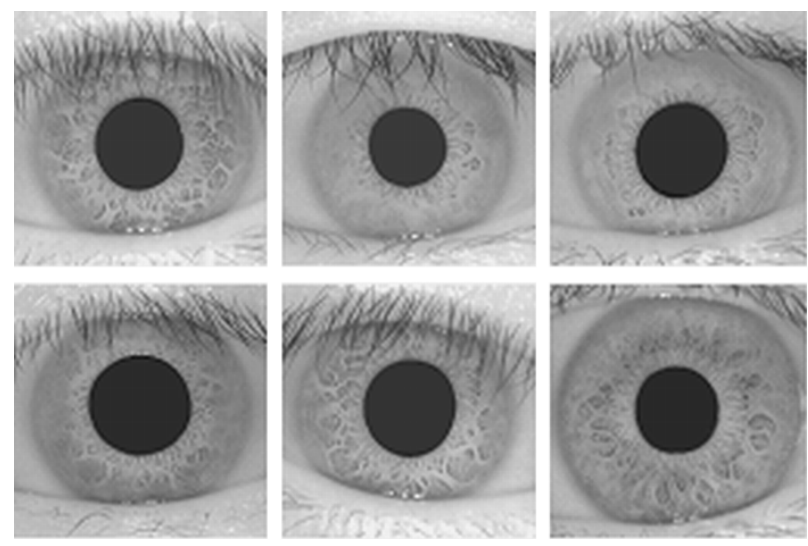

Fig. 1. Example of iris images from the CASIA iris image database [31].

the importance of phase for the perception of visual features. So the task of iris feature extraction is a process of phase demodulation. Regarding the multiscale two-dimensional (2-D) Gabor wavelets as the carrier waves, Daugman [6], [7] extracted the phase measures as the iris feature. The phase is coarsely quantized to four values and the iris code is 256 bytes long. Then the dissimilarity between the input iris image and the registered template can be easily determined by the Hamming distance between their IrisCodes. Sanchez-Reillo and Sanchez-Avila [8] demonstrated Daugman's idea with the intention to change the computation platform from PC to the embedded system. Based on the AM--FM image model [26], Tisse et al. [9] encoded the emergent frequency and instantaneous phase as iris features, which also needs a procedure of demodulation. Because phase congruency is a robust local image feature and can be approximated by a local energy model, Huang et al. [10] adopted a bank of Log-Gabor filters to represent local orientation characteristics of the iris.

2) Zero-crossings representation [11]-[13]: The zero-crossings of wavelet transform provide meaningful information of image structures [27]. In order to save computational cost, Boles and Boashash [11] calculated only a one-dimensional wavelet transform's zero-crossings over concentric circles on the iris. Then two different dissimilarity functions were employed for matching. Similarly, [12] also presented a zero-crossing representation of iris patterns, and the matching results based on three different distance metrics (Euclidean distance, Hamming distance, and direct distance associated with zero-crossing points) were reported. In [13], although the Haar wavelet frame decomposition method was used to extract the local features of iris pattern, in essence the result is a sort of zero-crossing description because at last the wavelet frame coefficients were encoded to $0 \mathrm{~s}$ or $255 \mathrm{~s}$ based on the threshold zero. Different from other iris recognition methods, this method [13] used the geometric moments of the decomposition result as an index to select a set of candidates in the database for further fine matching.

3) Texture analysis [14]-[23]: Naturally, random iris pattern can be seen as texture, so many well-developed texture analysis methods can be adapted to recognize the iris. In our early works [14], [15], multichannel Gabor filters are used to extract the iris features. from Gabor filters, a bank of circular symmetric filters was designed to capture the discriminating information along the angular direction of the iris image [16], [17]. Wildes 
et al. [18], [19] represented the iris pattern using a four-level Laplacian pyramid and the quality of matching was determined by the normalized correlation results between the acquired iris image and the stored template. In [20], Kumar et al. proposed an iris verification diagram by correlation filters. Because the directional feature is insensitive to contrast and illumination differences in an image or between images, Park et al. [21] decomposed the iris image into eight directional subband outputs using a directional filter bank. Lim et al. [22] applied a 2-D Haar wavelet transform four times to decompose the iris image and constructed a compact code from the high-frequency coefficients. The idea of Bae et al.'s algorithm [23] is to construct an optimal basis of iris signals using independent component analysis, and then each iris image can be represented by the coefficients of its projection on such a basis.

4) Local intensity variation [5], [24]: For a transient signal, the local variations denote its most important properties. In our recent works, the iris recognition algorithms based on characterizing local variations of image structures obtained encouraging results. Using a class of quadratic spline wavelets, Tan et al. [25] recorded the position sequence of local sharp variation points as the iris feature, which achieved high performance in both speed and accuracy. In [24], Tan et al. characterized the local intensity variations by Gaussian--Hermite moments.

Compared with the approaches based on texture analysis, the iris recognition methods using phase, zero-crossing, or local variation information pay more attention to the micro details or local features in an image. Based on our comparative study [25], the methods using local features generally have better performance than the texture analysis-based methods because the texture features are incapable of precisely capturing such fine spatial changes of the iris. As the texture is a regional property, this conclusion is straightforward. Considering the iris pattern as a random signal, the state-of-the-art local feature-based methods [6], [13], [25] had achieved high recognition accuracy by a test of statistical independence. But on the other hand, the measurements from minutiae are easily affected by noises, such as occlusions by eyelids and eyelashes, localization error, nonlinear iris deformations, etc., which greatly limits the system's accuracy. In our experiments [5], about $90 \%$ of the false nonmatches are due to these types of noises. Noisy as these iris images are, if we have a simple visual examination of any two images (Fig. 1), we can confidently determine whether they are from the same source or not. The reason is that the human vision procedure is coarse to fine; namely, the macrostructures are observed at first, when global information (such as the shape measurement of the blocks of interest) also has a powerful discriminating ability. Furthermore, if the global features are based on the physical properties of the iris image structure, such as the moments of the segmented blocks in an image, and an elastic matching diagram is applied in recognition, the global feature is expected to overcome the limitations of local feature based classifiers (LFC), i.e., sensitivity to photometric and geometric distortions. Therefore, it is necessary to complement the local features with global features to achieve the best possible recognition accuracy. Of course many well-developed classifier combination methods, such as the Neyman-Pearson rule, the weighted sum rule, Fisher discriminant analysis, etc. may be used. But the interest here is to achieve the trade-off between accuracy and efficiency, which is crucial to practical application. Thus, a cascading strategy is proposed in this paper. The basic idea of this technique is to construct a two-stage classification system with a reject option. The LFC is implemented first and the GFC (global feature based classifier) is seldom consulted unless the LFC is uncertain of its result, which is determined by the matching score between the input iris and the stored template. When the score is near the decision boundary of LFC and the input is assumed as gen-

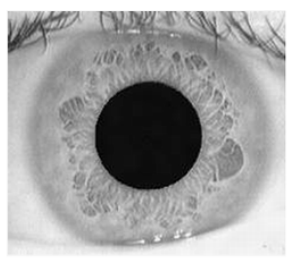

(a)

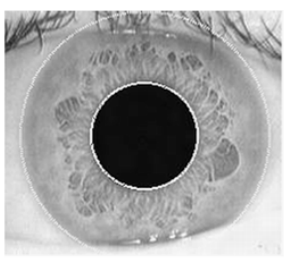

(b)

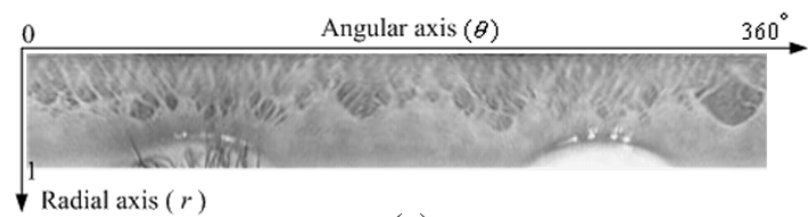

(c)

Fig. 2. Preprocessing of iris image: (a) original image; (b) result of iris localization; (c) normalized iris image.

uine, noisy iris images are usually involved in matching. Compared with LFC, the GFC is capable of recognizing noisy iris images. So the integrated LFC-GFC system is more accurate than the single classifier. Because the large majority of recognition tasks can be handled by LFC, the system's real-time performance is not essentially affected by the added GFC.

The remainder of this paper is organized as follows. Section II describes an elastic blob matching algorithm which attempts to establish the global correspondence between two iris images by the moments of the blobs. The multistage combination architecture will be introduced in Section III. Section IV provides the experimental results prior to conclusions in Section V.

\section{Moment-Based IrIS Blob Matching}

A typical iris recognition system includes localization, normalization, feature extraction, and matching. Fig. 2 illustrates the preprocessing step involving localization and normalization. The focus of this paper is pattern representation and matching. The details of preprocessing can be found in [6], [17] and [19].

Based on our observations, the block pattern in an iris image is very informative for iris matching. The objective of blob matching is to find the spatial correspondences between the blocks in the input iris image and that in the stored model, and to quantitatively assess their similarity based on the number of matched block pairs. If each block is denoted by its gravity center, then our task is a point pattern matching problem. It is well known that fingerprint minutiae matching is essentially a typical point set matching problem. Using ideas of alignment-based fingerprint minutiae matching [28], the whole process of iris blob matching is described in the following.

\section{A. Blocks of Interest Segmentation}

Before block pattern matching, the blocks of interest (BOI) should be isolated from the normalized iris image. In general, image segmentation techniques utilize intensity's homogeneity or discontinuity to subdivide an image into meaningful objects. The similarity of pixels in an iris BOI usually can not be guaranteed; thus the sharp variation of intensity along the boundary of each BOI provides more important information for segmentation. Because the zero-crossings of wavelet transform often indicate the location of sharp variation points [27], the boundary of a BOI can be efficiently detected after dyadic wavelet transform (DWT) performed on the input data [Fig. 3(b)]. With local processing of edge linking, the closed-boundary regions are labeled. Because the pixels in the region of a BOI always have lower intensity 
than others, the block is labeled as foreground if the result of DWT at its region is negative.

\section{B. Block Pattern Representation}

In the coordinate system shown in Fig. 2(c), each BOI is represented by a 2-D function $f(r, \theta)$, which is defined as follows:

$$
f(r, \theta)=\left\{\begin{array}{ll}
1, & (r, \theta) \in \mathrm{BOI} \\
0, & (r, \theta) \notin \mathrm{BOI}
\end{array} .\right.
$$

Then each BOI's centroid coordinates $(R, \bar{\theta})$ and area (Area), and the second order central moments (Moment $R$, Moment $\theta$ ) are recorded as its attributes. In fact, the five features can all be represented using geometric moments

$$
\begin{aligned}
R & =\frac{m_{10}}{m_{00}}=\frac{\iint r^{1} \theta^{0} f(r, \theta) d r d \theta}{\iint r^{0} \theta^{0} f(r, \theta) d r d \theta} \\
\bar{\theta} & =\frac{m_{01}}{m_{00}}=\frac{\iint r^{0} \theta^{1} f(r, \theta) d r d \theta}{\iint r^{0} \theta^{0} f(r, \theta) d r d \theta} \\
\text { Area } & =m_{00}=\iint r^{0} \theta^{0} f(r, \theta) d r d \theta \\
\text { Moment } R & =\mu_{20}=\iint(r-R)^{2}(\theta-\bar{\theta})^{0} f(r, \theta) d r d \theta \\
\text { Moment } \theta & =\mu_{02}=\iint(r-R)^{0}(\theta-\bar{\theta})^{2} f(r, \theta) d r d \theta .
\end{aligned}
$$

Generally, there are about one hundred BOIs in an iris image. So each iris image can be represented with a block set $\left\{\left(R_{i}, \theta_{i}\right.\right.$, Area $_{i}$, Moment $R_{i}$, Moment $\left.\left.\theta_{i}\right) \mid i=1,2, \ldots, N\right\}$, where $N$ denotes the total number of BOIs in the image.

\section{Alignment of Two Block Patterns}

After the iris localization and normalization shown in Fig. 2, the iris image achieves translation and scale invariant. However, the rotation difference has not been removed. So the rotation parameter between the two block patterns must be found first. Fortunately, the gravity centers of BOIs provide a good source of control points because they are stable under random noise. At first all corresponding block pairs of two iris patterns can be identified according to the following four criteria:

$$
\begin{aligned}
\left|R_{1}-R_{2}\right| & \leq T_{R} \\
\frac{\mid \text { Area }_{1}-\text { Area }_{2} \mid}{\min \left(\text { Area }_{1}, \text { Area }_{2}\right)} & \leq T_{A} \\
\frac{\mid \text { Moment } R_{1}-\text { Moment } R_{2} \mid}{\min \left(\text { Moment } R_{1}, \text { Moment } R_{2}\right)} & \leq T_{M R} \\
\frac{\mid \text { Moment } \theta_{1}-\text { Moment } \theta_{2} \mid}{\min \left(\text { Moment } \theta_{1}, \text { Moment } \theta_{2}\right)} & \leq T_{M \theta}
\end{aligned}
$$

where the variables with subscript " 1 " denote the features of the input block pattern and " 2 " represents those in the stored block pattern. The four predefined thresholds $T_{R}, T_{A}, T_{M R}$, and $T_{M \theta}$ construct the elastic bounding box. Pairs of corresponding blocks are supposed as the origins in their own images respectively, so blocks of other pairs should have relative angles ranging from $0^{\circ}$ to $360^{\circ}$ with respect to their reference blocks circularly. In each coordinate system pair established by corresponding block pairs, the number of block pairs which have similar relative $\theta$ location is counted. After all iterations, the rotation parameter can be computed from the optimal coordinate system pair with maximum matching count $N_{m}$. Then a representation invariant to translation, scale, and rotation is obtained after all BOIs' $\theta$ coordinates are updated in the aligned coordinate system.

\section{Similarity Assessment}

The registration of block patterns is based only on one to one correspondence, but it is possible that one block is physically divided into

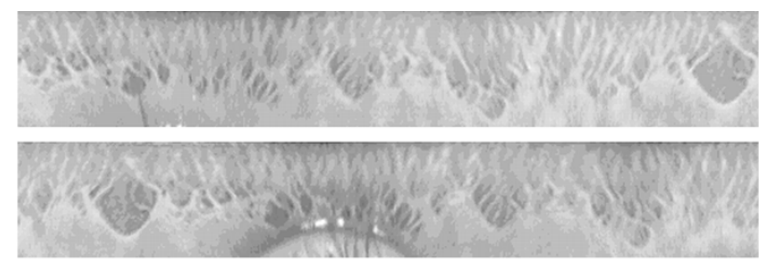

(a)

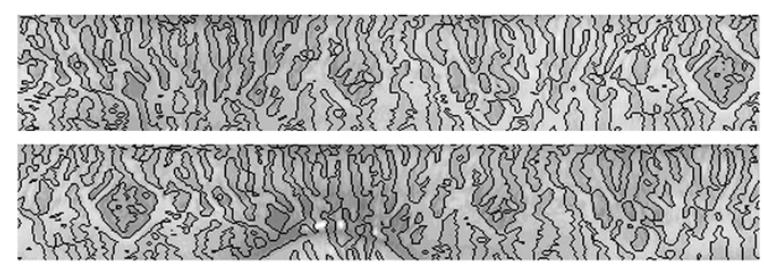

(b)

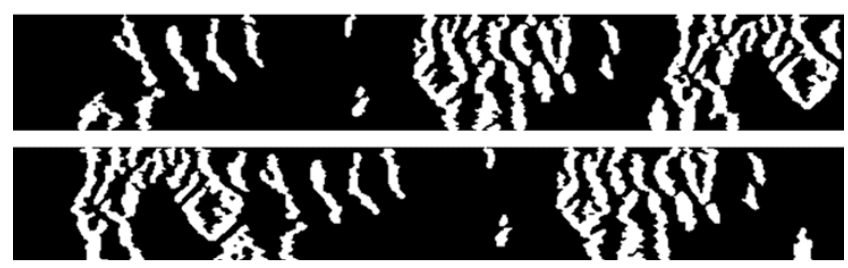

(c)

Fig. 3. Moment-based iris blob matching process: (a) two normalized iris images from same eye; (b) segmentation results (the overlaid contours denote zero-crossings of DWT); (c) the matched BOIs.

several smaller regions during segmentation. So if the mapping relationship between $M$ and $N$ blocks can be found, our result is more reasonable although it is a difficult problem. Fortunately, the proposed representation of block patterns facilitates this work because if $k$ blocks are assumed to be one, the feature of the unified larger block can be derived from the features of its components

$$
\begin{aligned}
R & =\frac{\sum_{i=1}^{k} R_{i} \text { Area }_{i}}{\sum_{i=1}^{k} \operatorname{Area}_{i}} \\
\theta & =\frac{\sum_{i=1}^{k} \theta_{i} \text { Area }_{i}}{\sum_{i=1}^{k} \operatorname{Area}_{i}} \\
\text { Area } & =\sum_{i=1}^{k} \operatorname{Area}_{i} \\
\text { Moment } R & =\sum_{i=1}^{k} \operatorname{Moment}_{i}+\sum_{i=1}^{k} \operatorname{Area}_{i}\left(R_{i}-R\right)^{2} \\
\text { Moment } \theta & =\sum_{i=1}^{k} \operatorname{Moment}_{i}+\sum_{i=1}^{k} \operatorname{Area}_{i}\left(\theta_{i}-\theta\right)^{2}
\end{aligned}
$$

where $\quad\left(R_{i}, \theta_{i}\right.$, Area $_{i}$, Moment $R_{i}$, Moment $\left.\theta_{i}\right)(i=1,2, \ldots, k)$ denotes the feature of the $i$ th block and $(R, \theta$, Area, MomentR, Moment $\theta$ ) is the feature of the unified one. At last, a quantitative matching score of the two iris block patterns is defined as

$$
M S=\min \left(\frac{M_{1}}{N_{1}}, \frac{M_{2}}{N_{2}}\right)
$$

where $M$ denotes the number of matched blocks and $N$ denotes the total number of blocks in an iris block pattern. The two iris block patterns are differentiated by the subscripts " 1 " and " 2 ."

\section{CASCADING ARChITECTURE FOR ACCURATE IRIS RECOGNITION}

An iris recognition system is usually deployed in a high security access control situation where false acceptance is strictly controlled by 


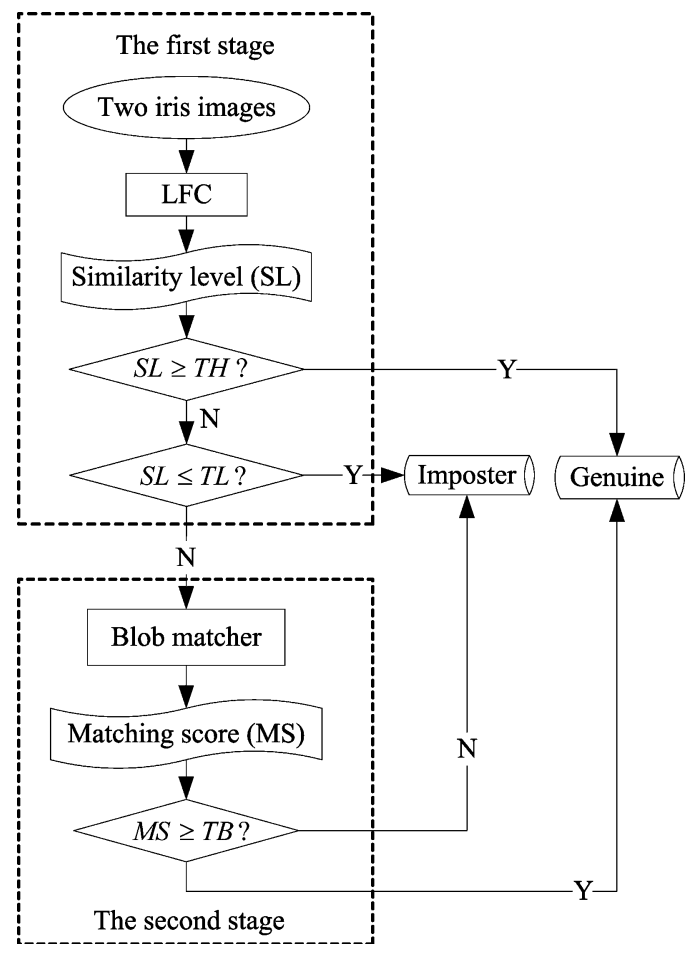

Fig. 4. Cascading scheme for iris recognition.

requiring a high matching score. The hypothesis of this kind of application is that we are imposters unless we have provided enough evidence to verify who we are. In real-world iris recognition scenarios, genuine subjects are easily rejected if the local feature-based approaches meet noises, which is the motivation of our current work. In this paper, we aim to reduce the system's false reject rate (FRR) when the system is required to operate at a low false accept rate (FAR). For this purpose, the cascading scheme is illustrated in Fig. 4.

This is a typical two-stage pattern classification system with a reject option whose theoretic foundation has been well built by Pudil et al. [29] and Kaynak et al. [30]. In the framework, the condition to evoke the second session is

$$
T L<S L<T H
$$

where $T L$ is defined via $\mathrm{P}(S L<T L \mid$ Imposter $)=0.9999$ and $T H$ is defined by $\mathrm{P}(S L<T H \mid$ Imposter $)=0.999999 . T L$ and $T H$ correspond to FAR $0.01 \%$ and $0.0001 \%$, respectively, and can be learned from the distribution of interclass comparisons. In practice, when the similarity level is larger than $T H$ we often consider that the input biometric pattern is from a genuine person confidently, and when the similarity level is less than $T L$ the subject is regarded as an imposter without further consideration. We found that the majority of falsely rejections' matching scores fall into the interval between $T L$ and $T H$. So the second session is introduced to give these falsely rejected subjects one more chance to provide alternative evidence to verify their identities.

\section{EXPERIMENTAL RESULTS}

\section{A. Database}

With a homemade iris imaging device, we have created an iris image database, the CASIA Iris Image Database [31]. This database has been shared worldwide for research purposes. The database includes 2255 iris image sequences from 306 different eyes (hence, 306 different classes) of 213 subjects. To satisfy the requirement of using images captured in different stages for training and testing,

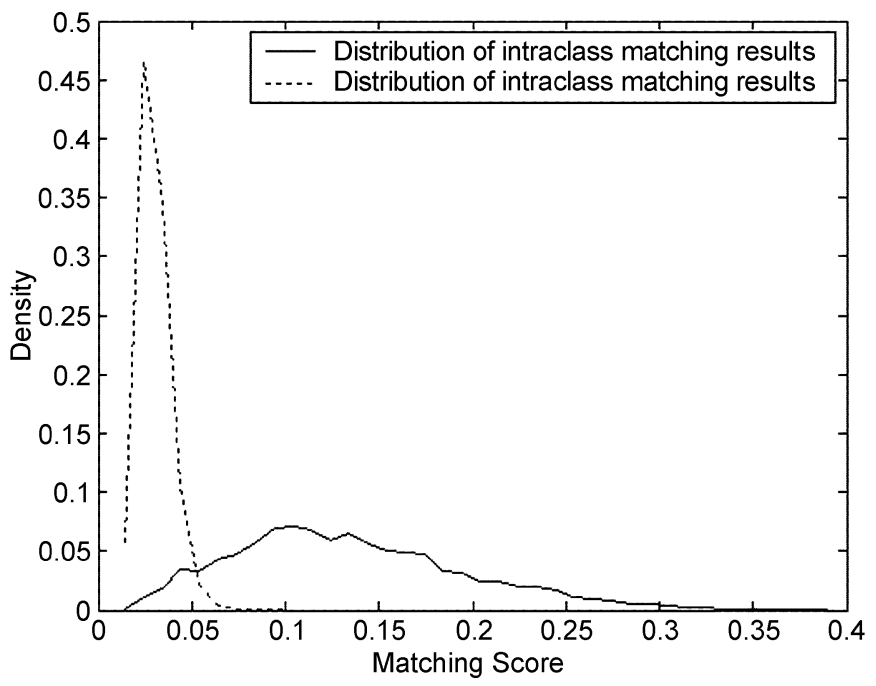

(a)

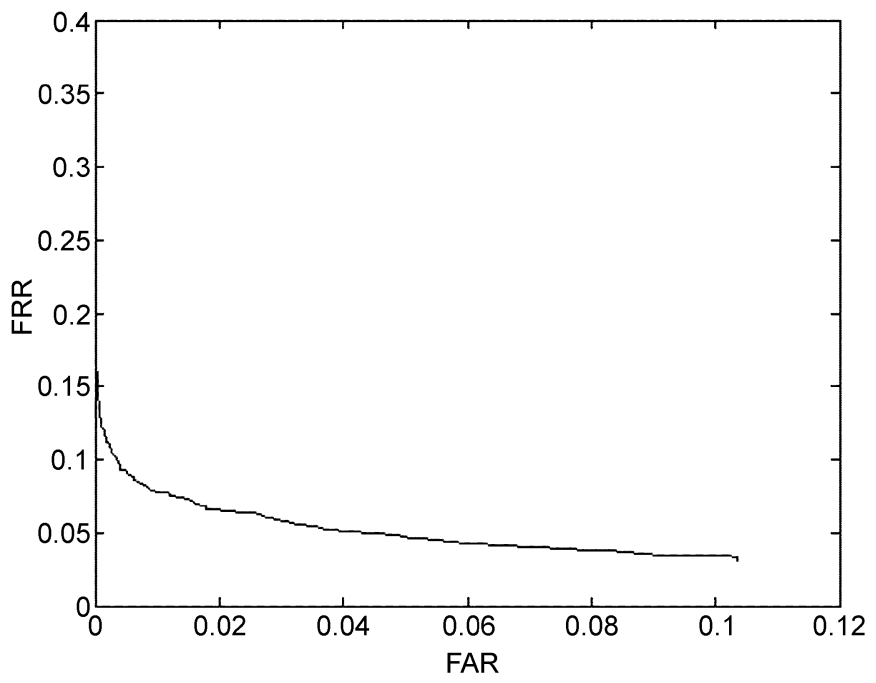

(b)

Fig. 5. Performance of moment-based blob matching algorithm: (a) distribution of intraclass and interclass matching scores; (b) ROC curve.

respectively, 100 taken at an earlier time are not used in the experiments. The images are separated into two sets: a training set of 918 template sequences (three sequences per eye) and a testing set of 1237 sequences of all people. For the images of the same eye, the time interval between the samples in the training set and the testing set is more than one month and the longest interval is about six months. The format of the data is 8-bits gray level image with resolution $320 \times 280$. Ten images in each sequence are automatically selected by an image quality assessment algorithm [17], but there are still some poor quality iris images used. In order to test our scheme in a low failure to enrollment rate (FTE) situation, we randomly select an image from the ten qualified images of each sequence to construct a dataset. And all comparisons in verification mode are made between data collected at two different times. So there are totally $918 \times 1237=1135566$ comparisons between the test image and the model data, including 3711 intraclass comparisons and 1131855 interclass comparisons.

\section{B. Discriminating Power of Blob Pattern Representation}

In order to evaluate the performance of the proposed iris blob matching algorithm, the distribution of matching scores on the dataset is shown in Fig. 5(a) and the receiver operating characteristic (ROC) 


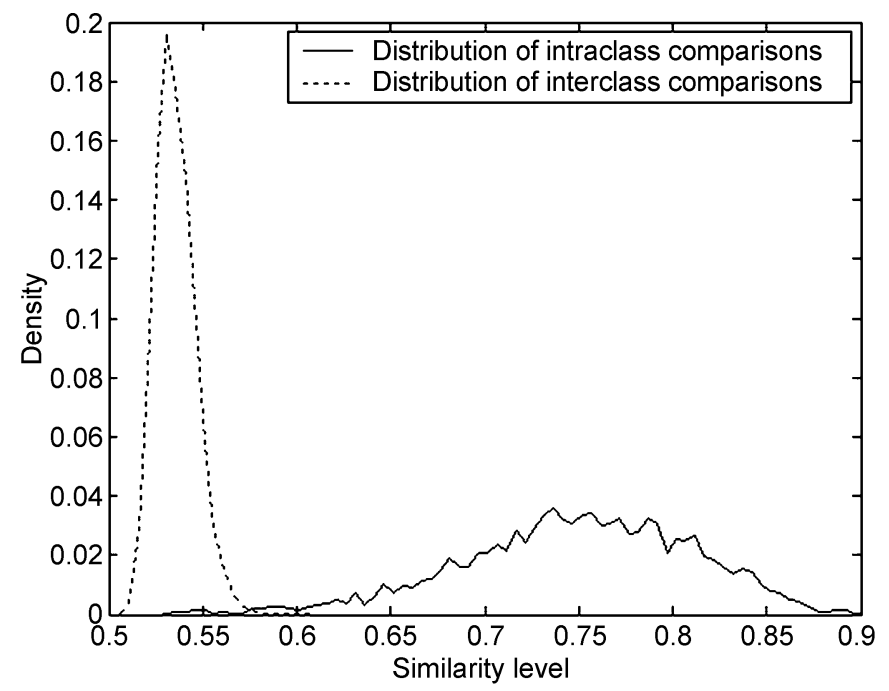

(a)

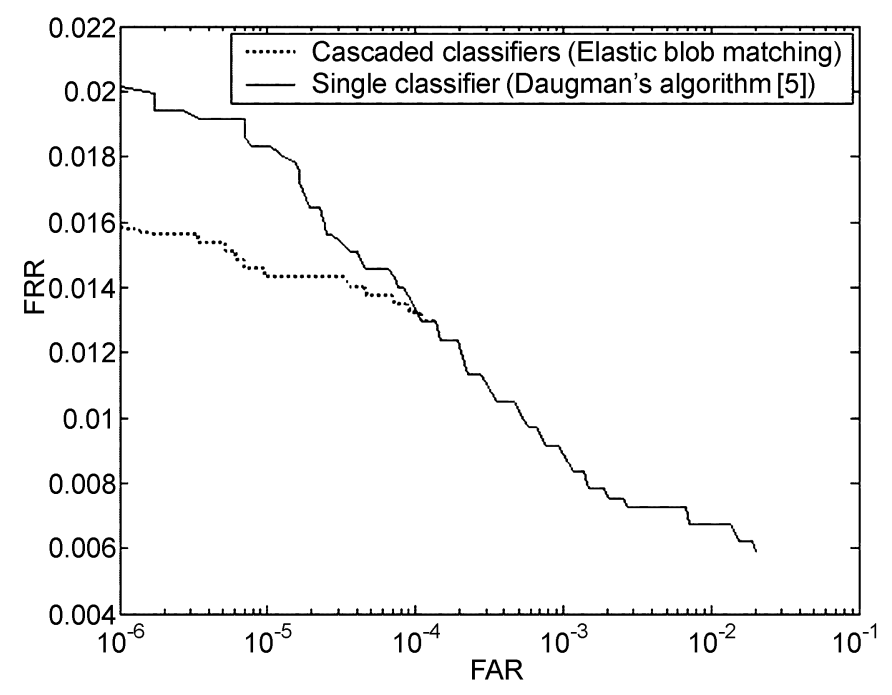

(b)

Fig. 6. Recognition performance comparisons between LFC (Daugman's method) and the cascading system: (a) distribution of genuine and imposter matching scores of Daugman's algorithm; (b) ROC curves of different classification approaches.

curve, a plot of the FRR against the FAR under different threshold values, is drawn in Fig. 5(b).

Because the precise localization of BOI can not be guaranteed in all iris images, the blob matching method does not perform as well as the state-of-the-art LFC [5], [6], [13]. But it is obvious in Fig. 5(a) that the matching score needed for confirmation of a genuine subject is very low (about six matched block pairs, which is small compared with the approximately one hundred blocks in an image), indicating that the blob matching method is robust against local noises to some extent. For LFC, if half of the iris image region is smeared it almost can not verify the genuine subject, but the blob matcher still has the possibility to search the credible evidence in the small region to authenticate the identity of the input image. In addition, the elastic matching strategy makes it accommodate localization error, distortion, and occlusions of eyelids or eyelashes. Therefore, the GFC is good at dealing with poor quality iris images compared with the LFC.

\section{Performance Evaluation of Cascaded Classifiers}

Both Daugman's algorithm [6] and Noh's method [13] are typical LFC. To test the recognition performance of the cascading scheme, they

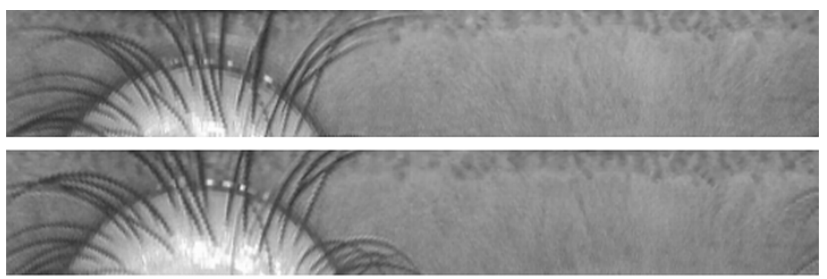

(a)

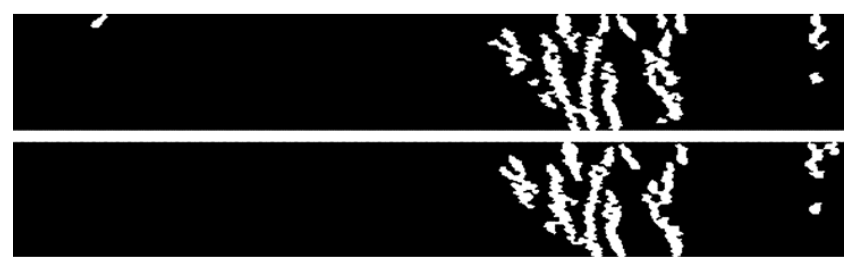

(b)

Fig. 7. An example where the GFC is more robust than the LFC. (a) Two normalized iris images from the same eye; the matching score of Daugman's method is 0.587 . (b) The matched BOIs by the blob matching algorithm; the blob matching score is 0.18 .

are combined with the GFC, respectively. It should be pointed out that the programs of both Daugman's method and Noh's system are coded following their publications [6], [13] and the length of their IrisCodes is 256 bytes and 144 bytes, respectively. Without strict image quality control or further manual rejection of poor-quality images, the performance of their algorithms in our dataset is worse than that reported in their publications.

The intercomparison and intracomparison results in the benchmark based on Daugman's method are illustrated in Fig. 6(a). According to the cascading diagram in Section III, the blob matching algorithm is called when the output of Daugman's algorithm satisfying

$$
0.5850<S L<0.6039
$$

where $S L=1-$ Hamming Distance, and 0.5850 corresponds to $T L$ and 0.6039 denotes $T H$. Both the thresholds are set based on the distribution of the interclass matching scores [Fig. 6(a)]. The ROC curves of both the single classifier and cascading system are demonstrated in Fig. 6(b) for comparison. We can see that when $10^{-6} \leq \mathrm{FAR} \leq 10^{-4}$ the cascading system achieves a lower FRR, when the system's operation state (FAR/FRR) is controlled by the criterion of the GFC. The two curves overlay when FAR $>10^{-4}$. The comparison result also proves that the blob matcher is more robust than LFC in the noisy environment.

One example is shown in Fig. 7 which is a false rejection by the LFC but correctly accepted by the GFC. Because of the occlusions of eyelids and eyelashes, a test of statistical independence [6] of the two images is passed. But the blob matching score is high enough to determine they are from the same eye.

Similar results of the Noh's system [13] are demonstrated in Fig. 8.

Based on the image quality, the iris data involved in recognition can be divided into two parts: good quality or poor quality (see Fig. 9). Through image quality assessment, most of the iris images qualified for recognition are really good quality and suitable for classification by LFC. But in a low FTE application, a few poor quality iris images are also used for recognition. Then the GFC is better than the LFC. This conclusion can be derived from the above experimental results.

According to the two kinds of iris images mentioned in Fig. 9, there are four possible classification strategies in iris recognition (Table I). So it is a perfect iris recognition system if the good-quality iris images are recognized by the LFC and the poor quality images are handled by the GFC. The proposed cascading scheme attempts to achieve this objective. In our case, the poor quality iris images are roughly identified by measuring whether the matching score of the LFC is near the classifier's decision boundary because the typical intraclass matching 


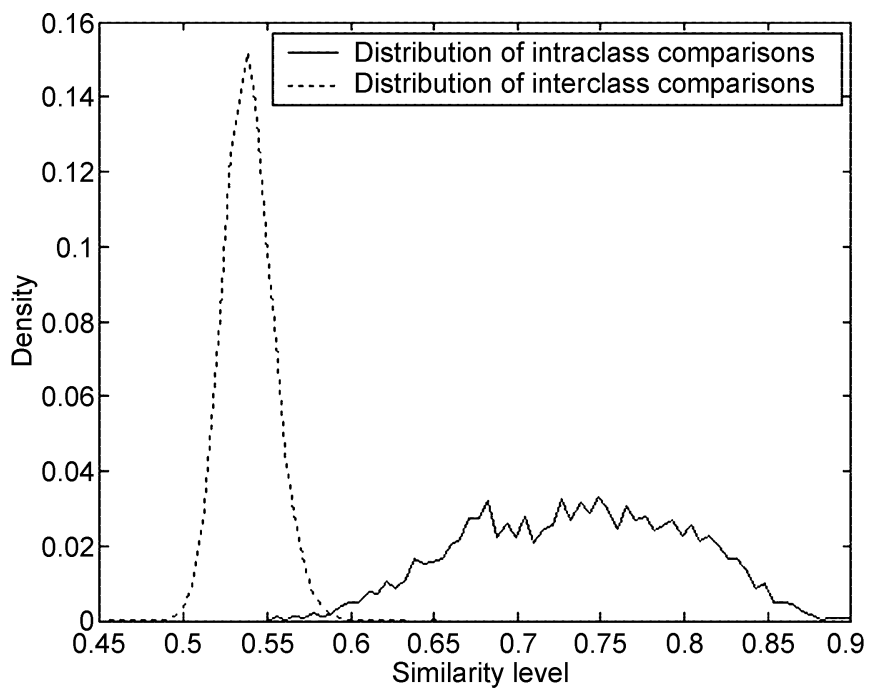

(a)

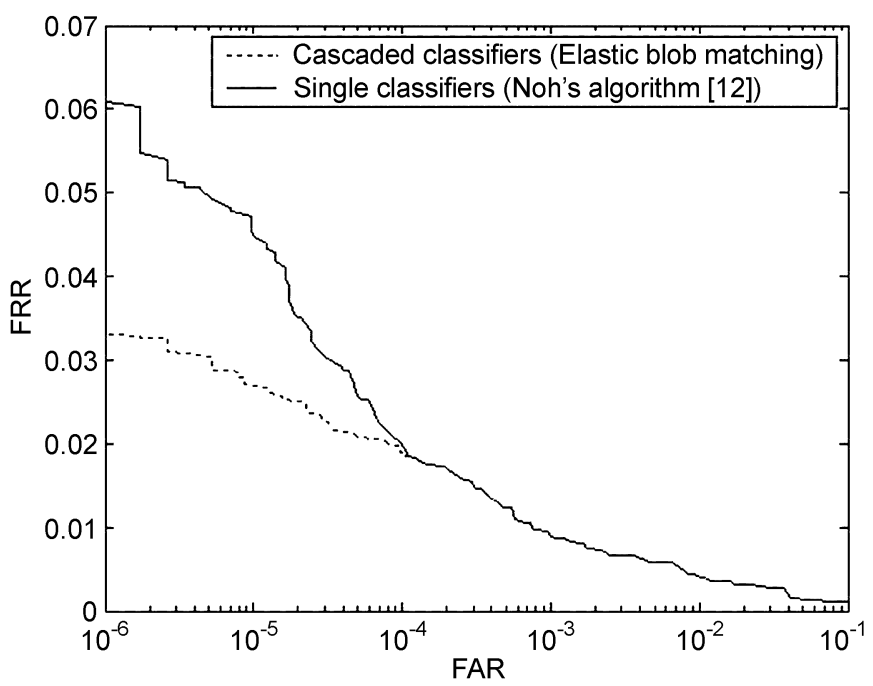

(b)

Fig. 8. Recognition performance comparisons between LFC and the cascading system (Noh's method). (a) Distribution of genuine and imposter matching scores of Noh's algorithm (use only the local feature). (b) ROC curves of different classification approaches.

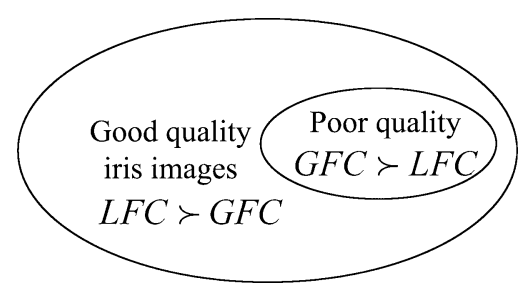

Fig. 9. Two kinds of iris images. The " $\succ$ " denotes "better than"; LFC and GFC have their own expertise for recognizing different quality iris images, i.e., they are image-quality dependent.

score is usually much higher than the decision boundary. In this sense, the LFC also acts as another iris image quality classifier. Therefore the cascading system outperforms the individual classifiers in terms of accuracy.

The computational cost of GFC is about two times of Daugman's algorithm, but its probability to be used is only about $1 \%$ empirically. So for Daugman's system, the average time cost only increases $2 \%$.
TABLE I

ReCOGNItion Results of Four Possible Classification Strategies

\begin{tabular}{c|c|c|c}
\hline $\begin{array}{c}\text { Classification } \\
\text { strategies }\end{array}$ & $\begin{array}{c}\text { Good quality } \\
\text { iris images }\end{array}$ & $\begin{array}{c}\text { Poor quality } \\
\text { iris images }\end{array}$ & $\begin{array}{c}\text { Recognition } \\
\text { result }\end{array}$ \\
\hline LFC+GFC & LFC & GFC & Best \\
\hline $\begin{array}{c}\text { Individual } \\
\text { classifier (LFC) }\end{array}$ & LFC & LFC & Better \\
\hline $\begin{array}{c}\text { Individual } \\
\text { classifier (GFC) }\end{array}$ & GFC & GFC & Good \\
\hline GFC+LFC & GFC & LFC & Worse \\
\hline
\end{tabular}

For Noh's algorithm, this number is about 3\%. Therefore, the computational performance of the overall algorithm is not significantly affected.

\section{CONCLUSION}

In this paper, a novel iris classifier combination scheme cascading the matching algorithms based on different representations is proposed. Based on the knowledge of the classifiers' expertise in discriminating different qualities of iris images, an LFC is replaced with a GFC to recognize noisy iris signals. If the slightly increased computational cost is negligible, this scheme is deserved. This idea is also applicable in other biometric systems.

Another contribution of this paper is that a novel moment-based iris blob matching algorithm is proposed, which can register two intraclass iris images with subpixel accuracy and measure their similarity. Note that almost all the existing iris recognition methods [6]-[17], [18]-[24] obtain only coarse rotation invariance. Although the experimental results show that the blob matching algorithm alone is not promising for iris recognition, it has its own specialties for discriminating noisy iris images.

Our further work is to incorporate the matched blocks' area information to improve the GFC's recognition rate. The indexing and retrieval of a large scale iris image database has never been addressed before although it is a very important issue in large population human identification. Almost all the existing iris recognition methods model the randomness of iris patterns using local statistical features, which are not suitable for database indexing. However, the moments of iris blob are good shape indices for database retrieval. This should be an interesting research topic.

\section{ACKNOWLEDGMENT}

The authors would like to thank the anonymous referees for their thorough reviews and constructive comments. Their thanks also go to those who have contributed to the establishment of the CASIA Iris Image Database.

\section{REFERENCES}

[1] A. K. Jain, R. M. Bolle, and S. Pankanti, Eds., Biometrics: Personal Identification in Networked Society. Norwell, MA: Kluwer, Jan. 1999.

[2] D. Zhang, Automated Biometrics: Technologies and Systems. Norwell, MA: Kluwer, May 2000.

[3] T. Mansfield, G. Kelly, D. Chandler, and J. Kane, "Biometric product testing final report, Issue 1.0," National Physical Laboratory of UK, Teddington, Middlesex, 2001.

[4] Iridian Technologies. Web siteAvailable: http://www.iridian.com/solutions.php [Online]

[5] L. Ma, T. Tan, Y. Wang, and D. Zhang, "Efficient iris recognition by characterizing key local variations," IEEE Trans. Image Process, vol. 13, pp. 739-750, Jun. 2004.

[6] J. Daugman, "High confidence visual recognition of persons by a test of statistical independence," IEEE Trans. Pattern Anal. Mach. Intell., vol. 15, no. 11, pp. 1148-1161, Nov. 1993. 
[7] _ " "Statistical richness of visual phase information: Update on recognizing persons by iris patterns," Int. J. Comput. Vision, vol. 45, no. 1, pp. 25-38, 2001.

[8] R. Sanchez-Reillo and C. Sanchez-Avila, "Iris recognition with low template size," in Proc. Int. Conf. Audio- and Video-Based Biometric Person Authentication, 2001, pp. 324-329.

[9] C. Tisse, L. Martin, L. Torres, and M. Robert, "Person identification technique using human iris recognition," in Proc. Vision Interface, 2002, pp. 294-299.

[10] J. Huang, L. Ma, Y. Wang, and T. Tan, "Iris recognition based on local orientation description," in Proc. 6th Asian Conf. Computer Vision, vol. II, 2004, pp. 954-959.

[11] W. W. Boles and B. Boashash, "A human identification technique using images of the iris and wavelet transform," IEEE Trans. Signal Process., vol. 46, no. 4, pp. 1185-1188, Apr. 1998.

[12] C. Sanchez-Avila, R. Sanchez-Reillo, and D. de Martin-Roche, "Iris-based biometric recognition using dyadic wavelet transform," IEEE Aerosp. Electron. Syst. Mag., vol. 17, pp. 3-6, Oct. 2002.

[13] S. Noh, K. Bae, and J. Kim, "A novel method to extract features for iris recognition system," in Proc. 4th Int. Conf. Audio- and Video-Based Biometric Person Authentication, 2003, pp. 838-844.

[14] Y. Zhu, T. Tan, and Y. Wang, "Biometric personal identification based on iris patterns," in Proc. 15th Int. Conf. Pattern Recognition, vol. II, 2000, pp. 805-808.

[15] L. Ma, Y. Wang, and T. Tan, "Iris recognition based on multichannel Gabor filtering," in Proc. 5th Asian Conf. Computer Vision, vol. I, 2002, pp. 279-283.

[16] - "Iris recognition using circular symmetric filters," in Proc. 16th Int. Conf. Pattern Recognition, vol. II, 2002, pp. 414-417.

[17] L. Ma, T. Tan, Y. Wang, and D. Zhang, "Personal identification based on iris texture analysis," IEEE Trans. Pattern Anal. Mach. Intell., vol. 25, no. 12 , pp. $1519-1533$, Dec. 2003

[18] R. P. Wildes, J. C. Asmuth, G. L. Green, S. C. Hsu, R. J. Kolczynski, J. R. Matey, and S. E. McBride, "A machine-vision system for iris recognition," Mach. Vision Applicat., vol. 9, pp. 1-8, 1996.

[19] R. P. Wildes, "Iris recognition: An emerging biometric technology," Proc. IEEE, vol. 85, no. 9, pp. 1348-1363, Sep. 1997.

[20] B. Kumar, C. Xie, and J. Thornton, "Iris verification using correlation filters," in Proc. 4th Int. Conf. Audio- and Video-Based Biometric Person Authentication, 2003, pp. 697-705.

[21] C. Park, J. Lee, M. Smith, and K. Park, "Iris-based personal authentication using a normalized directional energy feature," in Proc. 4th Int. Conf. Audio- and Video-Based Biometric Person Authentication, 2003, pp. 224-232.

[22] S. Lim, K. Lee, O. Byeon, and T. Kim, "Efficient iris recognition through improvement of feature vector and classifier," ETRI J., vol. 23, no. 2, pp. 61-70, 2001

[23] K. Bae, S. Noh, and J. Kim, "Iris feature extraction using independent component analysis," in Proc. 4th Int. Conf. Audio- and Video-Based Biometric Person Authentication, 2003, pp. 838-844.

[24] L. Ma, T. Tan, D. Zhang, and Y. Wang, "Local intensity variation analysis for iris recognition," Pattern Recognition, vol. 37, no. 6, pp. 1287-1298, 2005.

[25] A. V. Oppenheim and J. S. Lim, "The importance of phase in signals," Proc. IEEE, vol. 69, pp. 529-541, 1981.

[26] J. Havlicek, "AM-FM image models," Ph.D. dissertation, Department of Electrical and Computer Engineering, Univ. Texas, Austin, 2002.

[27] S. Mallat, "Zero-crossings of a wavelet transform," IEEE Trans. Inf. Theory, vol. 37, no. 4, pp. 1019-1033, Apr. 1992.

[28] A. K. Jain, L. Hong, and R. Bolle, "On-line fingerprint verification," IEEE Trans. Pattern Anal. Mach. Intell., vol. 19, no. 4, pp. 302-314, Apr. 1997.

[29] P. Pudil, J. Novovicova, S. Blaha, and J. Kittler, "Multistage pattern recognition with rejection option," in Proc. 11th Int. Conf. Pattern Recognition, vol. B, 1992, pp. 92-95.

[30] C. Kaynak and E. Alpaydin, "Multistage cascading of multiple classifiers: One man's noise is another man's data," in Proc. 17th Int. Conf. Mach. Learni., 2000, pp. 455-462.

[31]. Center for Biometrics and Security Research. CASIA Iris Image Database [Online] Available: http://www.sinobiometrics.com/casiairis.htm

\section{A Real-Time Focusing Algorithm for Iris Recognition Camera}

\author{
Kang Ryoung Park and Jaihie Kim
}

\begin{abstract}
For fast iris recognition, it is very important to capture the user's focused eye image at fast speed. Previous researchers have used the focusing method which has been applied to general landscape scenes without considering the characteristics of the iris image. So, they take much focusing time, especially in the case of the user with glasses. To overcome such problems, we propose a new iris image acquisition method to capture focused eye images at very fast speed based on corneal specular reflection. Experimental results show that the focusing time for both users with and without glasses averages $480 \mathrm{~ms}$, and we conclude that our method can be used for the real-time iris recognition camera.
\end{abstract}

Index Terms - Iris image acquisition, iris recognition, specular reflection.

\section{INTRODUCTION}

With the increasing need for higher security levels, biometric systems have been widely used with many applications. Among them, the iris recognition system has been in the limelight for high-security biometric applications [1], [5]. For fast iris recognition, it is essential to capture a focused eye image at fast speed. If not, the total recognition time is increased, causing severe inconvenience to the user. Previous researchers [2]-[4], [8]-[15], used the focusing method which has been applied to general scenery (landscape or photographic) without considering the characteristics of iris image. In detail, they use the focusing algorithm that calculates the gray level difference between the spacing pixels. That is, if the input image is defocused, then the difference value between the pixels is low. If the image is highly clear, the difference value is high. From that, they move the focusing lens to the direction of making the difference value the highest. However, their method can provide the wrong focusing value in the case of iris images. Especially in the case of users with glasses, if the lens is positioned for focusing the glass surface or the glass frame, a scratch on the glass surface or the glass frame may make that the difference value of the spacing pixel its highest. In general, the distance between glasses and the iris is more than $1 \mathrm{~cm}$ and the input iris image remains blurred unless the focus lens does not move. Due to those problems, researchers [16] have used the method of checking the pixel difference in the region of specular reflection on a cornea. However, they use only one illuminator for checking focus value and iris recognition. In such a case, the focus checking is impossible when specular reflection, which happens on the surface of glasses, hides that on a cornea. In addition, when many specular reflections happen from the scratch on the glass surface, it is very difficult to detect the genuine specular reflection on a cornea by that method [16]. To overcome such problems, we propose a new focusing method to capture a user's focused eye image at very fast speed using corneal specular reflections with an On/Off scheme of dual illuminators. Base on such a focusing algorithm, we implemented a real-time iris recognition camera.

Manuscript received December 12, 2003; revised May 20, 2004. This work was supported by Korea Science and Engineering Foundation (KOSEF) through Biometrics Engineering Research Center (BERC) at Yonsei University. This paper was recommended by Guest Editor D. Zhang.

K. R. Park is with the Division of Media Technology, SangMyung University, Seoul 110-743, Republic of Korea (e-mail: parkgr@smu.ac.kr).

J. Kim is with the Department of Electrical and Electronic Engineering, University, Seoul 120-749, Republic of Korea (e-mail: jhkim@ yonsei. ac.kr).

Digital Object Identifier 10.1109/TSMCC.2005.848168 